\title{
Restriction Fragment Length Polymorphism of the C1 Inhibitor Gene in Hereditary Angioneurotic Edema
}

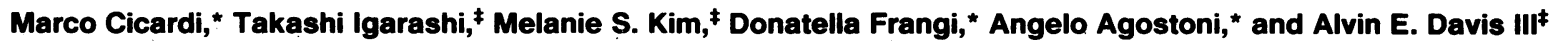 \\ *Cattedra di Clinica Medica Universita di Milano, Ospedale S. Paolo, Milan, Italy; and ${ }^{\ddagger}$ Department of Pediatrics, Harvard Medical \\ School, and Divisions of Immunology and Nephrology, The Children's Hospital, Boston, Massachusetts 02115
}

\begin{abstract}
Hereditary angioneurotic edema (HANE) results from the deficiency of the inhibitor of the first component of human complement (C1-INH). It is inherited as an autosomal dominant trait. Heterogeneity of this defect has been shown at the protein and mRNA level. Southern blot analysis of genomic DNA was performed after digestion with six different restriction endonucleases in 24 families affected with type 1 HANE (low antigenic and functional C1-INH levels) and five with type 2 (low functional C1-INH levels and normal or elevated levels of dysmorphic C1-INH). Blots were hybridized with a C1-INH cDNA probe of 1,227 bp. With one enzyme (Pst I), two different patterns of restriction fragment length polymorphism (RFLP) were detected. One was present in one kindred with type 1 HANE and the other appeared the same in one type 1 and in one type 2 family, thus indicating that each RFLP resulted from a different mutation. Analysis of a total of 34 members of these three families suggested that the polymorphisms are tightly linked to the mutation responsible for the disease. Using a 170-bp probe we showed that the three different mutations leading to these polymorphisms are located in the same region of the $\mathrm{C} 1-\mathrm{INH}$ gene. These data suggest that different mutations in the same region of the $\mathrm{C1}$-INH gene are responsible for C1-INH deficiency in these families. Most of these mutations are probably point mutations or other "minor" defects and do not appear to be due to major deletions or rearrangements.
\end{abstract}

\section{Introduction}

Hereditary angioneurotic edema (HANE) ${ }^{1}$ is due to the deficiency of the inhibitor of the first component of human complement (C1-INH) and is inherited as an autosomal dominant trait (1). The disease is characterized by episodic localized

Portions of this work were presented at the 1987 National Meeting of the American Society for Clinical Investigation (1987. Clin. Res. 35:606 Abstr.).

Address reprint requests to Dr. A. E. Davis, The Children's Hospital, Division of Immunology, 300 Longwood Ave., Boston, MA 02115. 1987.

Received for publication 28 May 1987 and in revised form 27 July

1. Abbreviations used in this paper: HANE, hereditary angioneurotic edema; C1-INH, $\mathrm{Cl}$ inhibitor; RFLP, restriction fragment length polymorphism.

J. Clin. Invest.

(C) The American Society for Clinical Investigation, Inc. $0021-9738 / 87 / 12 / 1640 / 04 \$ 2.00$

Volume 80, December 1987, 1640-1643 swelling of the subcutaneous tissue, or of the gastrointestinal or laryngeal mucosa (2-4). Symptoms can be controlled by therapy with androgen derivatives (5), and attacks can be reversed by the infusion of $\mathrm{Cl}$-INH plasma concentrate (6). Heterogeneity of the defect has been shown at the protein and mRNA level (7-9). Most individuals with HANE have decreased serum concentration of both antigenic and functional $\mathrm{C1}$ INH; mononuclear phagocytes from these patients have levels of C1-INH mRNA that are approximately half of normal (type 1 HANE). Type 2 HANE, which comprises $15 \%$ of affected families, is characterized by the presence of normal or increased concentrations in serum of a mutant C1-INH. Analysis of the C1-INH gene in normal individuals has revealed a restriction fragment length polymorphism (RFLP) using the restriction endonuclease HgiA I (10). Southern blot analysis from 10 patients failed to detect any $\operatorname{RFLP}(10,11)$. However, one report has indicated that polymorphism of the C1-INH gene is relatively common among patients with HANE (12). In the data presented here, we have evaluated 29 HANE kindred for RFLPs. Among these families only 2 of 24 with type 1, and 1 of 5 with type 2 revealed RFLPs linked to C1-INH deficiency.

\section{Methods}

Patients. 34 subjects belonging to 24 families with type 1 HANE and 27 belonging to 5 families with type 2 were studied. Among the type 1 families, 21 were from Italy (all of Italian ancestry) and 3 were caucasian American (national origin not known). Two of the type 2 families were Italian and three were caucasian American (national origin not known). 18 normal volunteers were analyzed at the same time.

Preparation of human genomic DNA and Southern blotting. $30 \mathrm{ml}$ of blood were drawn into a syringe containing $1 \mathrm{ml}$ of $200 \mathrm{mM}$ EDTA, pH 8. The DNA was extracted from white blood cells, as previously described (13). DNA samples of 5-10 $\mu \mathrm{g}$ were digested with restriction enzymes at a concentration of $5 \mathrm{U} / \mu \mathrm{l}$ (New England Biolabs, Beverly, MA). DNA was separated by electrophoresis on $0.8 \%$ agarose gel (Ultrapure agarose; Bethesda Research Laboratories, Gaithersburg, MD), treated according to Wahl et al. (14), and blotted onto nitrocellulose (BA 85, Schleicher \& Shuell, Inc., Keene, NH) (15). Prehybridization and hybridization were carried out at $42^{\circ} \mathrm{C}$ in a mixture containing $50 \%$ formamide. The C1-INH probe used was a 1,227-bp cDNA (pC1-INH II6) that extended from nucleotide 554 to nucleotide 1,780 (numbered according to Bock et al. [10]). This probe was isolated from a human liver cDNA library cloned into the plasmid pAT 153 (16). Labeling of the probe was achieved by nick translation (Nick Translation Kit; (Bethesda Research Laboratories) using alpha $\left[{ }^{32} \mathrm{P}\right] \mathrm{dCTP}$ (New England Nuclear, Boston, MA). Hybridization patterns were visualized by autoradiography. As an alternative to radioactive label, some of the experiments were done by nick translation of the probe by biotin "dUTP (Bethesda Research Laboratories). Visualization was obtained by enzymatic reaction (Blue Gene Kit; Bethesda Research Laboratories). 

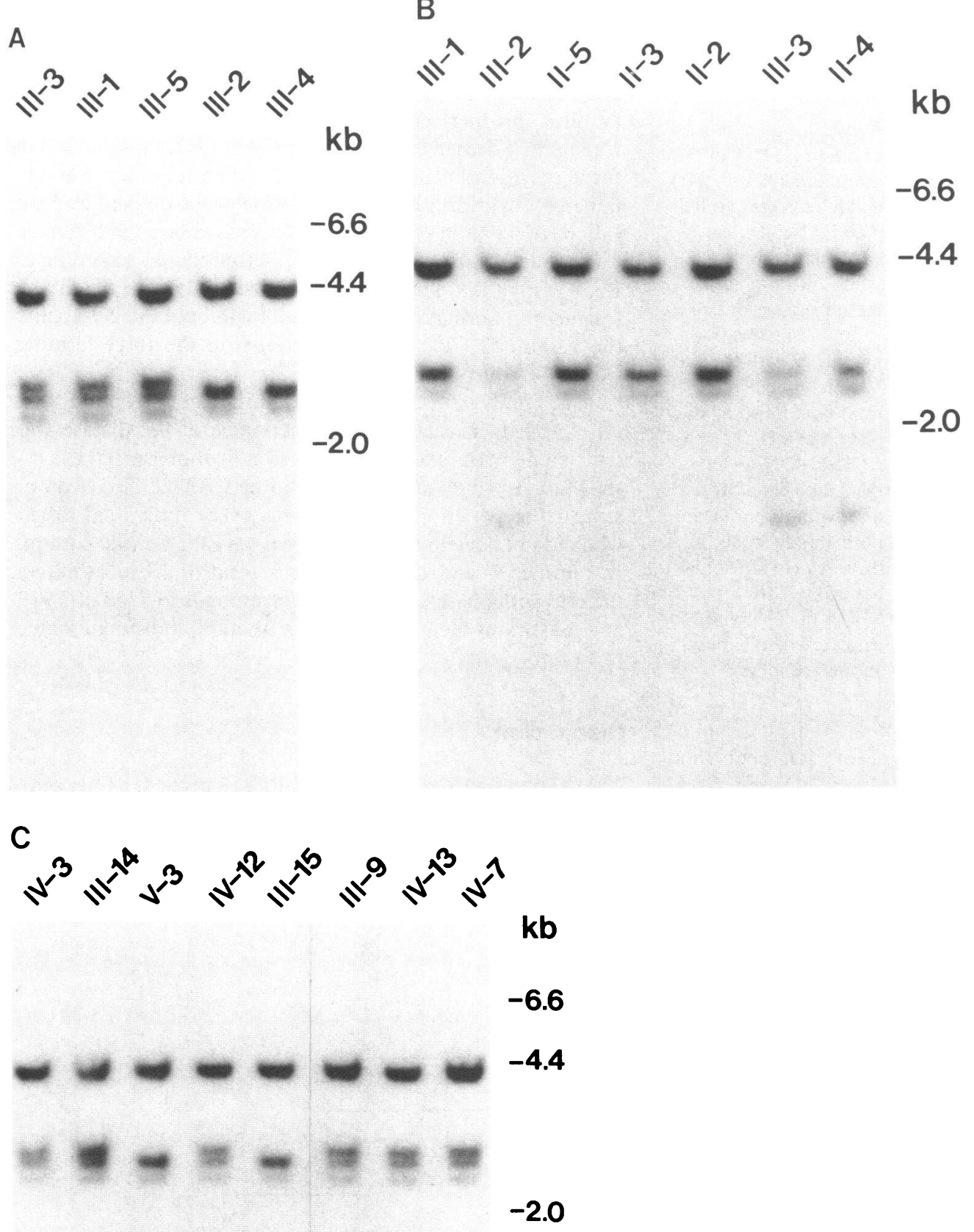

\section{Results}

Genomic DNA from 61 HANE patients and 18 normal individuals was subjected to Southern blot analysis after digestion with six different restriction endonucleases (Pst I, Hind III,
Figure 1. RFLP detected after Pst I digestion of genomic DNA in three different families affected with type 1 HANE $(A$ and $B)$ and type $2(C)$. The blots were hybridized with a 1,227-bp C1-INH cDNA probe. Numbers on each track indicate the subject according to fig. 3. Nonaffected members of these families show three major bands of 4.2, 2.9, and $2.7 \mathrm{~kb}$. Individuals with HANE in families $A$ and $C$ have an additional band of $3.1 \mathrm{~kb}$. In these subjects the $2.9-\mathrm{kb}$ normal band appears half the intensity of the same band in nonaffected individuals. In family $B$ the members with the disease have an additional band of $1.7 \mathrm{~kb}$. The $2.9-\mathrm{kb}$ normal band in these subjects is also half the intensity of the same band in nonaffected individuals.

Bam HI, Eco RI, Pvu II, Bgl II). Samples from patients and normal individuals revealed identical patterns with five of the six endonucleases. With Pst I, polymorphism was detected in three affected Italian kindred. Two of the families $(A$ and $B$ ) were affected with type 1 HANE; family $C$ had type 2 disease. 


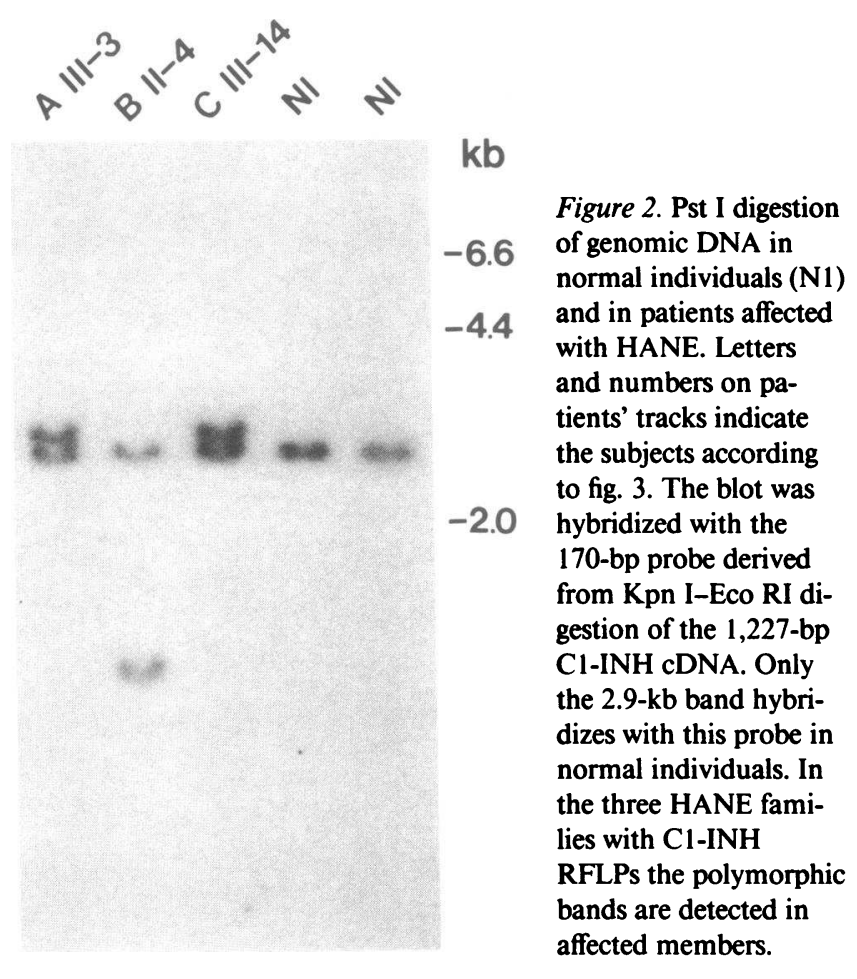

The "normal" pattern for Pst I consists of four fragments that hybridize with the probe, with sizes of $4.2,2.9,2.7$, and $<0.5$ kb (Fig. 1). The smallest fragment is not shown in the figures. Families $A$ and $C$ revealed, in addition to the normal pattern, a band of $3.1 \mathrm{~kb}$ (Fig. 1). In each of the affected individuals the 2.9-kb band appears to be present at one-half the intensity of the same band in the normal patterns. This suggests that the 3.1-kb fragment may result from a mutation affecting the 2.9$\mathrm{kb}$ fragment. Affected individuals in family $B$ have a $1.7-\mathrm{kb}$ band in addition to the normal pattern. As in the other two families, this fragment also very likely is related to the $2.9-\mathrm{kb}$ fragment. Further evidence that the mutation resulting in these polymorphisms involved the 2.9-kb fragment was obtained by hybridization with a 170-bp probe derived by Kpn I-Eco RI digestion of $\mathrm{pCI}$-INH II6. This probe extended from nucleotide 1,114 to nucleotide 1,284 (numbered according to Bock et al. [10]). This probe hybridized only with the 2.9-kb fragment in normal individuals, and with both the $2.9-\mathrm{kb}$ and the variant $(3.1$ or $1.7 \mathrm{~kb})$ fragments in the three families whose DNA is polymorphic (Fig. 2).

The family trees of these three families are shown in Fig. 3. As expected, in each family, inheritance of the disease was consistent with that of an autosomal dominant trait. As shown, every individual tested who had HANE also demonstrated the polymorphism, whereas every individual tested who did not have the disease showed only the normal pattern. In families $B$ and $C$, there were a total of 13 informative meioses with phase known. This corresponds to a lod of 3.913 at a maximum theta (recombinant fraction) indistinguishable from zero.

\section{Discussion}

The data presented here show that RFLPs in the C1-INH gene are unusual among patients with HANE. Southern blot analysis of DNA after digestion with different restriction endonucleases revealed polymorphism in only 3 of 29 families with HANE. This finding, together with previous data $(8,10)$, indi-
A

I

II

III

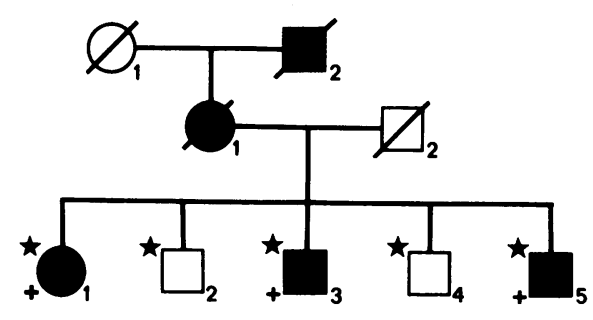

B

I

II

III

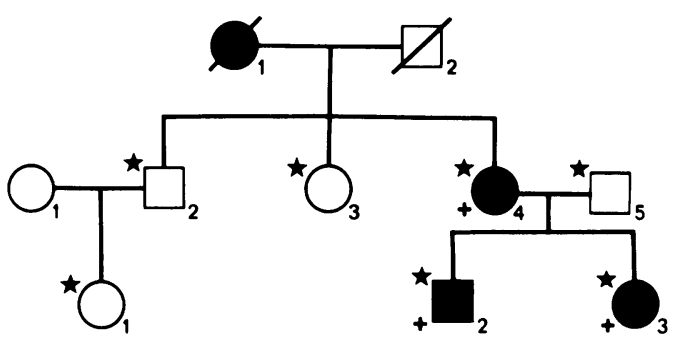

C

II

III

IV

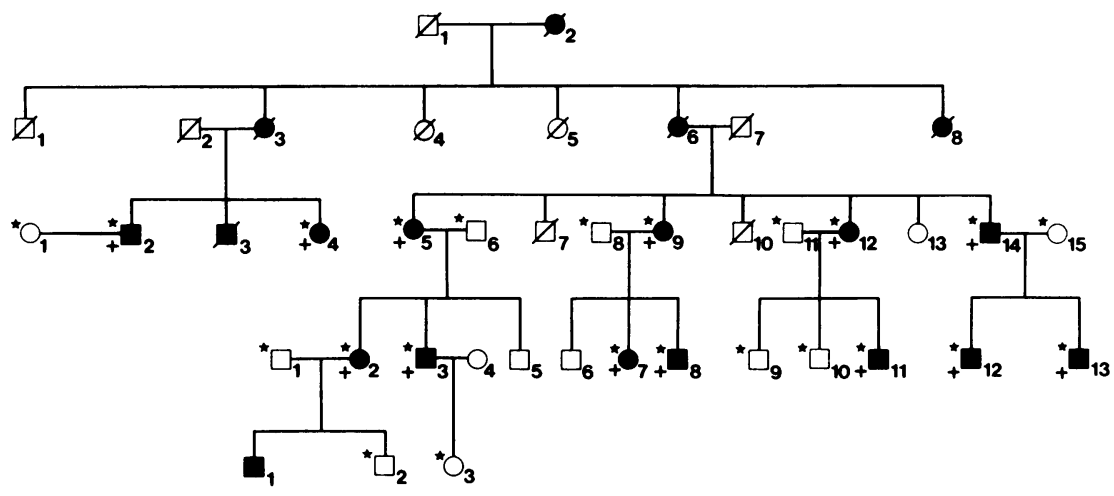

Figure 3. Genealogic trees of families affected with type 1 HANE ( $A$ and $B$ ) and type $2(C)$, carrying an RFLP of the C1-INH gene. Stars indicate individuals tested by Southern blot analysis. Diagonal lines indicate dead subjects. Dark symbols represent family members affected with HANE, and plus signs indicate individuals with an RFLP. 
cate that most mutations leading to $\mathrm{Cl}$-INH deficiency are not the result of major chromosomal rearrangements or deletions. Stoppa-Lyonnet et al. (12) found polymorphisms in four of seven families analyzed. This apparent difference in incidence of detected RFLPs may simply be due to the difference in the total number of kindred evaluated. It is also possible, as has been pointed out, that there may be different molecular genetic defects in different populations (17). Also, the observation that the polymorphisms were detected with only one restriction endonuclease indicates that even among these three families the molecular genetic defects are relatively minor. The most common abnormalities, therefore, probably are point mutations, or relatively small deletions or rearrangements. These results are in contrast with those of Stoppa-Lyonnet et al. (12), who detected polymorphic variants with several restriction endonucleases, suggesting major structural changes in the gene. The cDNA probe used in the studies described here was slightly larger and extended somewhat beyond each end of the cDNA used in their study $(12,18)$. It is therefore unlikely that their probe would detect variants that would be missed with the probe used here. Their findings, together with the findings presented here, further emphasize the heterogeneity of the molecular genetic defects observed in HANE $(7-9,12)$.

It seems likely that the polymorphisms detected in this study result from the mutations that are responsible for $\mathrm{Cl}$ INH deficiency. It is clear from the lod score and the fact that no recombinants were observed that the polymorphisms are tightly linked to the mutation responsible for the disease. Definitive proof of this conclusion will require sequence analysis of the genes from these patients. The two type 1 families (families $A$ and $B$ ), since they have different RFLPs must have different mutations. Family $C$ has an RFLP that appears the same as that in family $A$. However, these must result from different mutations since the patients in family $C$ synthesize a mutant protein (type $2 \mathrm{HANE}$ ), whereas the patients in family $A$ do not (type 1 HANE). Each of the polymorphic fragments was detectable with a 170-bp probe that encodes amino acids 338-394; the normal 2.9-kb fragment was also detectable with this probe. Thus, each of the three variants resulted from alterations within the same region of the gene. The relationship of these variants to the intron-exon structure of the gene cannot yet be defined. The region of the gene affected differs from the region affected in three of the four previously described patients with RFLPs. These findings, however, at least suggest the possibility that particular regions of the gene may be more subject to mutation.

\section{Acknowledgments}

We thank Dr. Fred S. Rosen, Dr. Chester A. Alper, and Dr. Michael Carroll for their helpful discussion during the course of these studies.

This work was supported by U. S. Public Health Service grants AM 34604 and HD 22082, by the March of Dimes Birth Defect Foundation grant 1-775, and by Consiglio Nazionale delle Ricerche Rome Special Project Basi Molecolari delle Malattie Ereditarie. This work was done during the tenure of an Established Investigatorship of the American Heart Association (Dr. Davis), and with funds contributed in part by the American Heart Association Massachusetts Affiliate, Inc.

\section{References}

1. Donaldson V. H., and R. R. Evans. 1963. A biochemical abnormality in hereditary angioneurotic edema. Am. J. Med. Sci. 35:37-44.

2. Cicardi M., L. Bergamaschini, B. Marasini, G. Boccassini, A. Tucci, and A. Agostoni. 1982. Hereditary angioedema: an appraisal of 104 cases. Am. J. Med. Sci. 284:2-9.

3. Brickman C. M., G. C. Tsokos, J. E. Balow, T. J. Lawley, M. Santaella, C. M. Hammer, and M. M. Frank. 1986. Immunoregulatory disorders associated with hereditary angioedema. I. Clinical Manifestations of autoimmune disease. J. Allergy Clin. Immunol. 77:749-757.

4. Brickman C. M., G. C. Tsokos, T. M. Chused, J. E. Balow, T. J. Lawley, M. Santaella, C. H. Hammer, G. F. Linton, and M. M. Frank. 1986. Immunoregulatory disorders associated with hereditary angioedema. II. Serologic and cellular abnormalities. J. Allergy Clin. Immunol. 77:758-767.

5. Gelfand J. A., R. J. Sherins, D. W. Alling, and M. M. Frank. 1976. Treatment of hereditary angioedema with danazol. Reversal of clinical and biochemical abnormalities. $N$. Engl. J. Med. 295:14441448.

6. Bergamaschini L., M. Cicardi, A. Tucci, M. Gardinali, D. Frangi, C. Valle, and A. Agostoni. C1 INH concentrate in the therapy of hereditary angioedema. 1983. Allergy (Copenh.). 38:81-84.

7. Rosen F. S., C. A. Alper, J. Pensky, M. R. Klemperer, and V. H. Donaldson. Genetically determined heterogeneity of the $\mathrm{Cl}$ esterase inhibitor in patients with hereditary angioneurotic edema. 1971. J. Clin. Invest. 50:2143-2149.

8. Gadek J. E., S. W. Hosea, J. A. Gelfand, and M. M. Frank. 1979. Response of variant angioedema phenotypes to danazol therapy. $J$. Clin. Invest. 64:280-286.

9. Cicardi M., T. Igarashi, F. S. Rosen, and A. E. Davis III. 1987. Molecular basis for the deficiency of complement 1 inhibitor in type 1 hereditary angioneurotic edema. J. Clin. Invest. 79:698-702.

10. Bock S. C., K. Skriver, E. Nielsen, M. C. Thogersen, B. Wiman, V. H. Donaldson, R. L. Eddy, J. Marrinan, E. Radziejewska, R. Huber, T. Shows, and S. Magnusson. 1986. Human Cl inhibitor: primary structure, cDNA cloning and chromosomal localization. Biochemistry. 25:4292-4301.

11. Davis A. E. III, A. S. Whitehead, R. A. Harrison, A. Dauphinais, G. A. P. Bruns, M Cicardi, and F. S. Rosen. 1986. Human Cl inhibitor: characterization of cDNA clones and localization of the gene to chromosome 11. Proc. Natl. Acad. Sci. USA 83:3161-3165.

12. Stoppa-Lyonnet D., M. Tosi, J. Laurent, A. Sobel, G. Lagrue, and T. Meo. 1987. Altered $\mathrm{Cl}$ inhibitor genes in type 1 hereditary angioedema. N. Engl. J. Med. 317:1-6.

13. Whitehead A. S., D. E. Woods, E. Fleishnick, J. E. Chin, E. J. Yunis, A. J. Katz, P. S. Gerald, C. A. Alper, and H. R. Colten. 1984. DNA polymorphism of the $\mathrm{C} 4$ genes: a new marker for analysis of the major histocompatibility complex. N. Engl. J. Med. 310:88-91.

14. Wahl G. M., M. Stern, and G. R. Stark. 1979. Efficient transfer of large DNA fragments from agarose gels to diazobenzoloxymethal paper and rapid hybridization using dextran sulfate. Proc. Natl. Acad. Sci. USA 76:3683-3687.

15. Southern E. 1975. Detection of specific sequence among DNA fragments separated by gel electrophoresis. J. Mol. Biol. 98:503-517.

16. Belt K. T., M. C. Carroll, and R. R. Porter. 1984. The structural basis of the multiple forms of human complement component $\mathrm{C} 4$. Cell. 36:173-180.

17. Colten H. R. 1987. Hereditary angioneurotic edema, 1887 to 1987. N. Engl. J. Med. 317:43-45.

18. Tosi M., C. Duponchel, P. Bourgarel, M. Colomb, and T. Meo. 1986. Molecular cloning of human $\mathrm{Cl}$ inhibitor: sequence homologies with alpha 1-antitrypsin and other members of the serpin superfamily. Gene (Amst.). 42:265-272. 